\title{
LA MEDITERRANEE OCCIDENTALE DEPUIS L'OLIGOCENE SCHEMA D'EVOLUTION *
}

\author{
Xavier LE PICHON, Guy PAUTOT, Jean-Marie AUZENDE et Jean-Louis OLIVET \\ Centre Océanologique de Bretagne, France
}

Reçu le 6 août 1971

\begin{abstract}
- The evolution of the Western Mediterranean basin during Tertiary time is discussed. It is proposed that it was created by a continental drift process during Middle Oligocene time, synchronously with the creation of the grabens over the continents. The significance of the Messinian evaporitic sedimentary episode is discussed in liaison with the problem of large scale subsidence which has been at least six kilometers since Oligocene time. -
\end{abstract}

\section{Introduction}

Deux problèmes majeurs marquent l'histoire cénozoïque des Alpes et de la Méditerranée occidentale: à l'Oligocène moyen, tandis que l'arc alpin vient d'être le siège d'une phase de compression paroxysmale apparemment dirigée vers le nord-nord-ouest, une phase de distension commence à créer immédiatement à l'ouest de cet arc une série de grabens approximativement alignés du nord au sud (fig. 1). A la fin du Miocène durant la période appelée Messinien ou Pontien (dans ce texte, nous considérons qu'il s'agit de la période comprise entre 9 et $6 \mathrm{~m}$.a.) une couche d'évaporites s'ajoute, dans le bassin algéro-provençal et la mer Tyrrhénienne, à l'épaisse série marine antérieure [1-3]. Or, cette période marque pour plusieurs auteurs [4-6], le début d'une phase tectonique générale, bien distincte de la tectonique alpine antérieure.

Dans ce travail, nour proposons de lier la phase de distension oligocène à la création du bassin algéroprovençal après l'épisode de compression maximum, et à partir de cette hypothèse nous discutons la signification de l'épisode Pontien. Nous n'aborderons pas le problème de la mer d'Alboran, ni celui du sillon qui la prolonge entre le sud du socle des Baléares et l'Afrique du Nord (fig. 1). La fig. 1 est un schéma synthétique

\footnotetext{
* Contribution no 66 du Groupe Scientifique du Centre Océanologique de Bretagne.
}

qui montre l'extension probable des blocs continentaux et de la couche d'évaporites d'après les travaux océanographiques du Centre Océanologique de Bretagne. Les critères utilisés pour déterminer l'extension des blocs continentaux sont la morphologie du socle révélée par la sismique réflexion et la nature des anomalies magnétiques. Remarquez que l'extension considérable des blocs continentaux amène à poser différemment les problèmes de dérive, puisqu'on distingue un large bassin triangulaire nord-Baléare et un étroit sillon nordafricain. Dans cet article, nous discutons surtout la genèse du bassin nord-Baléare.

\section{L'âge tertiaire du Bassin}

Les études de sismique réfraction $[7,8]$ et de magnétisme [9] suggèrent que la partie abyssale du bassin algéro-provençal est de nature océanique. L'âge et le mode de création de ce bassin sont controversés. Ainsi Glangeaud [10] a proposé une ouverture du bassin, contemporaine de la phase de distension triasicoliasique. Plusieurs auteurs ont fait l'hypothèse d'une dérive du bloc corse-sarde au Cénozoïque. Ryan [11] par exemple, la place à l'Eocène, Bourcart [12], Dubourdieu [13], Stanley et Mutti [14] et Pannekoek [15] la placent au Miocène.

Pour notre part, nous pensons qu'un âge préTertiaire du bassin algéro-provençal est exclu. 


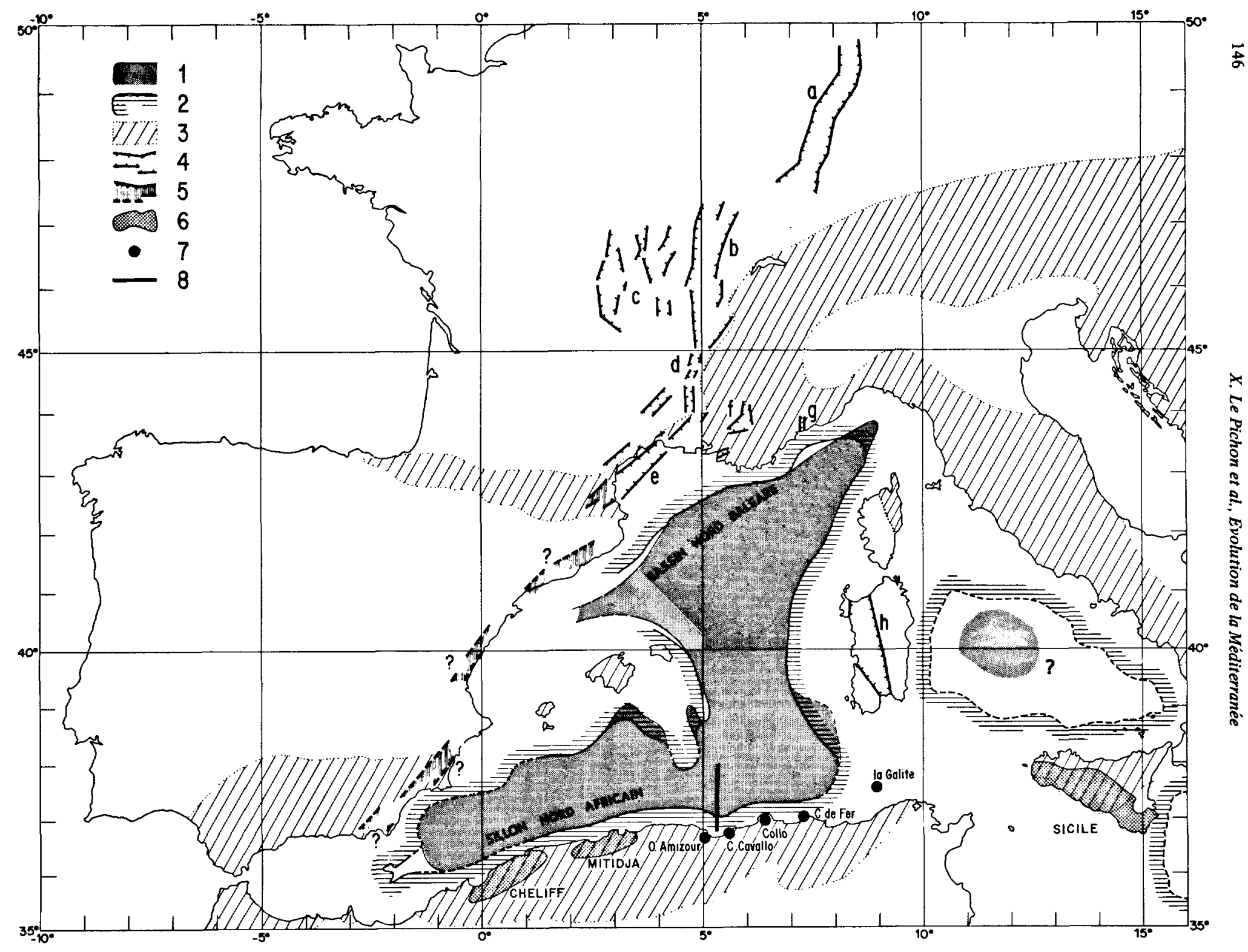

Fig 1. Diagramme structural composite de l'ensemble méditerranéen occidental. Remarquez en particulier que l'extension de la partie océanique probable du bassin est très limitée. (1) Extension de la couche évaporitique. (2) Extension probable du socle continental à partir des données de la sismique réflexion et du magnétisme. (3) Domaine affecté par les phases orogéniques tertiaires. (4) Fossés oligocènes. (5) Fossés Miocène supérieur et moyen. (6) Principaux bassins miocènes dans lesquels a été reconnue la couche de sel messinienne. (7) Profil présenté sur la fig. 2. 
En effet, la profondeur du socle magnétique [9] est de l'ordre de 8 à $10 \mathrm{~km}$ sous la surface de l'eau, dans la partie profonde du bassin. Ceci correspond au toit de la couche à $6,7 \mathrm{~km} / \mathrm{sec}$ révélée par la sismique réfraction $[7,8]$. On sait que l'épaisseur de la couche d'eau est d'environ $2,5 \mathrm{~km}$, celle du Plio-Quaternaire va de 0,5 à $1,5 \mathrm{~km}$, celle du sel messinien de 0,5 à 1 $\mathrm{km}$. C'est donc 3 à $6 \mathrm{~km}$ de sédiments qui se sont déposés avant le Messinien. On peut suivre sur certains profils $[1,2]$ des strates régulières jusqu' à 2 secondes sous les évaporites, soit au moins $3 \mathrm{~km}$. Nous observons donc la presque totalité du remplissage du bassin. Il est difficile d'y envisager la présence de sédiments mésozoïques sans admettre un taux de sédimentation très inférieur au taux moyen durant le Plio-Pleistocène ( 8 à $24 \mathrm{~cm}$ par mille ans) étant donné qu'il n'y a aucun indice de discordance importante qui pourrait être causée par une lacune de sédimentation. On ne peut donc lier l'ouverture du bassin à la phase de distension triasico-liasique [10].

Pour rendre compte d'observations sédimentologiques ou tectoniques, la présence d'un domaine continental émergé jusqu'au tertiaire à l'emplacement du domaine abyssal actuel, a souvent été proposée. Il s'agit tantôt du simple prolongement des chaînes actuelles, tantôt de l'hypothétique continent "Tyrrhénide" [16]. Par exemple, Kuenen et al. [17] et Stanley et Mutti [14] concluent de l'analyse des flyschs des Alpes Maritimes (d'âge Eocène supérieur et Oligocène basal) qu'une partie des sources d'apport devait se trouver entre la Corse et la Provence actuelle.

Si nous admettons l'hypothèse du déplacement mésozoique du bloc ibérique pour expliquer la formation du golfe de Gascogne [18, 19], nous devons admettre que la création du bassin occidental de la Méditerranée lui est postérieure. Il n'existe, en effet, dans ce bassin, aucune structure susceptible d'avoir absorbé un déplacement de cette importance. Le déplacement du bloc ibérique aurait été accompagné par celui du bloc corse-sarde et probablement par celui de l'ensemble italo-dinarique.

Enfin, la création du bassin est probablement postérieure aux phases de compression pyrénéoprovençale du Crétacé supérieur et de l'Eocène supérieur, car les plissements languedociens s'expliquent plus facilement avec la présence d'un bloc continental au sud.

\section{Hypothèse de l'âge d'ouverture Oligocène}

A l'Oligocène moyen, une phase de distension se marque, entre les Pyrénées et la Provence par la création de fossés (fig. 1) (fossés d'Alès, de Narbonne, du Bas-Rhône [20]) et par l'affaissement du golfe du Lion (Olivet, en préparation). A l'est se forment les fossés de Marseille et de Manosque. Une suite de fossés analogues s'alignent vers le Nord: couloir rhodanien, I imagnes, Bresse, fossé du Rhin. Une prolongation vers le nord (Vogelsberg, golfe du Kassel, Fjord d'Oslo) a été souvent proposée mais reste hypothétique. L'essentiel de la subsidence dans les fossés se produit au cours de l'Oligocène moyen pendant lequel se déposent de fortes épaisseurs de sédiments (souvent plusieurs milliers de metres) marins et saumatres avec intercalation de niveaux évaporitiques [20]. Il nous paraît donc probable que l'ouverture du bassin se soit faite durant cette phase de distension. En effet, les considérations développées précédemment ayant montré que le remplissage du bassin avait commencé longtemps avant le Messinien, mais après le Mésozoïque, cette phase de distension est la seule connue dans cette région dans cet intervalle de temps. L'identification des anomalies magnétiques dans l'Atlantique, au $\mathrm{N}$ et au $\mathrm{S}$ de la ligne des Açores, conduit également à admettre une phase de distension à cette époque [21] . Dans cette hypothèse, la création du bassin, par dérive relative de la Corse et de la Sardaigne, se serait accompagnée d'un soulèvement avec fracturation des marges, de chaque côté des rifts initiaux, suivi par une subsidence au fur at à mesure que le bassin s'élargissait par mise en place de croûte océanique $[24,25]$. Ces phénomènes de soulèvement et subsidence sont liés à des processus thermiques. Or, on sait que cette période, le long des marges actuelles de ce bassin, est une période de régression généralisée [22, 23].

Certains auteurs pensent que l'on retrouve des structures d'effondrement jusqu'au sud-est de l'Espagne (Fontbote, communication personnelle). Mais, dans cette région, ces structures datent du Miocène moyen et inférieur et pourraient peut-être marquer un âge plus tardif d'ouverture de cette partie de la Méditerranée (entre les Baléares et l'Espagne et en mer d'Alboran). 
S.

N.

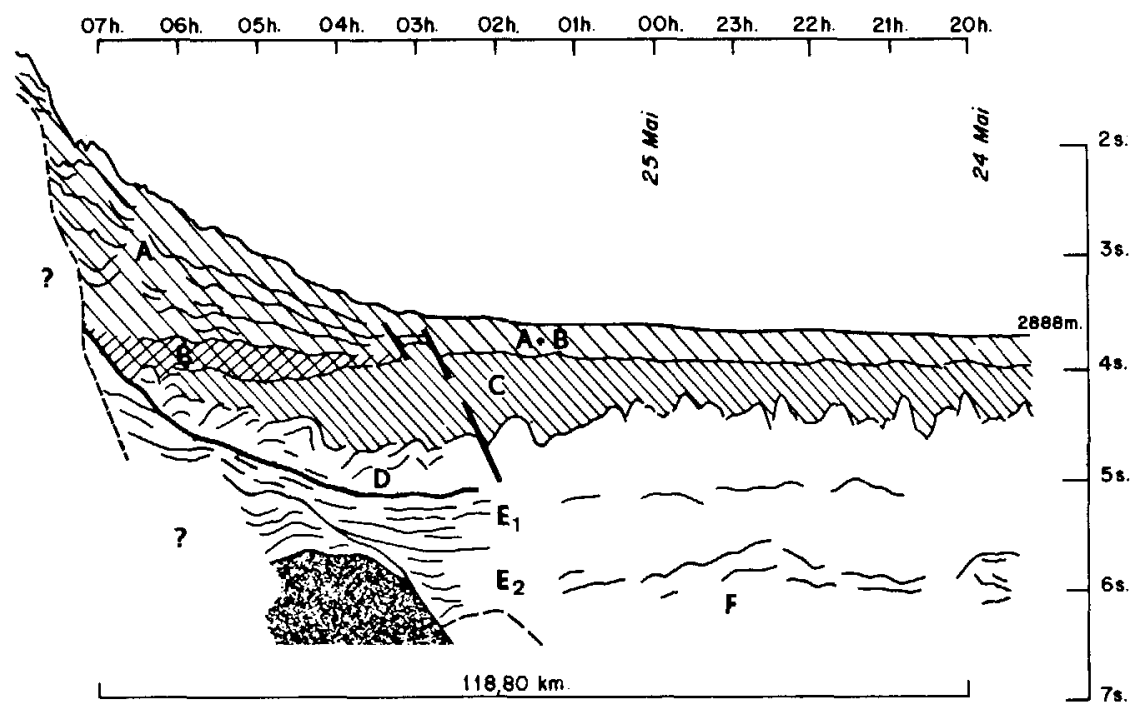

Fig. 2. Schéma interprétatif d'un profil Flexotir dans le golfe de Bougie (d'après Auzende et Pautot, 1970) (exagération verticale 12). En grisé: le substratum. A, B et C: sédiments post-messiniens. D: couche évaporitique. En trait épais: la surface pré-messinienne (pontienne) due à l'érosion sur la pente. $E_{1}, E_{2}$ et $F$ : sédiments anté-messiniens. La séries $E_{1}$ repose sur un lambeau de glissement alors que la série $E_{2}$ vient butter sur celui-ci. Le glissement serait donc synchrone du dépôt de $E_{2}$. On remarque que la série $\mathrm{F}$ est pratlquement horizontale jusqu’à au moins $60 \mathrm{~km}$ au nord de la pente.

\section{Le Miocène}

Une transgression marine importante succède à l'épisode de régression de l'Oligocène. Elle débute sur les marges à l'Aquitanien [26]. Cette transgression progresse de manière continue jusqu'au Tortonien (12 M.a.). Toutefois, durant la même époque (Miocène Inférieur et Moyen), les nappes de flyschs sont mises en place par gravité sur la bordure nordafricaine. De nombreux auteurs [27-29] pensent que les nappes de flyschs proviennent d'un "sillon" situé au nord de la marge continentale actuelle. Ceci impliquerait qu'au moins cette partie du bassin aurait été profondément affectée à cette époque, ayant subi un soulèvement de plusieurs kilomètres et une érosion très importante. Or, un examen des profils sismiques perpendiculaires à la marge nord-africaine ne permet pas de reconnaitre les traces de cet épisode tectonique dans le bassin actuel. La fig. 2, légèrement modifiée d'après Auzende et Pautot [30] montre que, sous la couche évaporitique messinienne [2] il existe une couche épaisse d'au moins $1500 \mathrm{~m}$ de sédiments horizontalement stratifiés et peu ou pas déformés. Cette couche, appelée E sur la figure, a une épaisseur constante sur plus de $100 \mathrm{~km}$ au nord de la marge. Donc si l'on admet des taux de sédimentation comparables aux taux actuels pour la période pré-Messinienne: 8 à $24 \mathrm{~cm}$ par mille ans qui, compte-tenu de la compaction, correspondent à 5 à $15 \mathrm{~cm} /$ mille ans, on est conduit à donner un âge Aquitanien à Oligocène Inférieur à la base de la couche E. Or, il semble qu'il existe audessous de cette couche d'autres niveaux sédimentaires horizontaux ( $F$ sur la figure). Il nous parait donc peu probable que les nappes de flyschs aient eu leur origine dans le bassin méditerranéen dont la configuration ne semble pas avoir notablement changé.depuis cette époque et ceci est en accord avec les hypothèses publiées récemment par Durand-Delga [31] et Wezel [32]. Nous ne pouvons pourtant pas exclure une création post-Miocène inférieur de la partie profonde du sillon nord-africain actuel, si l'on accepte des taux de sédimentation pré-messiniens supérieurs à $25 \mathrm{~cm}$ par 
mille ans et si on considère que la couche $F$ représente le socle. Auzende et Pautot [30] ont noté l'existence d'une certaine tectonique pré-Messinienne sur la marge continentale. Nous pensons que cette tectonique s'explique par des mouvements verticaux de la marge, peut-être liés à des variations eustatiques. En effet, il semble qu'il s'agisse essentiellement de glissements pré-messiniens suivis par une période d'érosion messinienne. En tout état de cause, cette tectonique de marge n'a pas été accompagnée d'une tectonique du bassin profond adjacent.

\section{Le Messinien et le Plio-Quaternaire}

La fin du Miocène (Messinien ou Pontien) est marquée sur tout le pourtour méditerranéen et alpin par le retrait de la mer de la plus grande partie du domaine continental actuel. Au même moment, dans toute la partie abyssale du bassin méditerranéen se dépose une épaisse série évaporitique $[1,2]$ dont la profondeur actuelle est d'environ $3500 \mathrm{~m}$, sauf sur certaines marges continentales où elle remonte jusqu'à $2500 \mathrm{~m}$, comme au sud des Baléares (Mauffret et al., en préparation et sur la marge ligure (Rehault et al., en préparation). On trouve apparemment une série évaporitique d'épaisseur comparable dans des bassins marginaux nord-africains comme ceux du Cheliff et de la Mitidja [33] et le bassin sicilien [34]. On sait que ces dernières régions ont été affectées par une tectonique verticale importante depuis le Messinien. Ainsi, les séries évaporitiques du bassin sicilien se trouvent à des altitudes de plus de $800 \mathrm{~m}$. On trouve également des évaporites messiniennes dans la région sud-espagnole, dans le bassin de l'Ebre, dans le delta du Rhône et en Toscane [15]. Ces évaporites sont en général recouvertes par des marnes bleues plaisanciennes ayant un faciès profond (au moins 500 à 1000 m) à Sélaciens et Téléostéens (Thaler et Cappetta, communication personnelle).

C'est durant le Messinien que la morphologie actuelle des pentes continentales semble avoir été modelée par un épisode d'émersion et d'érosion à laquelle on a attribué le creusement des canyons sous-marins dans les terrains antépliocènes $[4,35]$.

Dans le domaine alpin, les Apennins et la bordure africaine, la fin du Miocène (depuis 10 M.a.) est marquée par une phase de mouvements verticaux re- sponsables en particulier de la fermeture des détroits reliant le bassin méditerranéen à l'Atlantique (voir par exemple Geze [36] pour l'arc subalpin de Nice, Glangeaud [37] pour le Jura, Debelmas et Lemoine [38] pour le domaine alpin). On a généralement considéré cette phase comme une phase de distension, du fait en particulier de l'absence de tectonique tangentielle. Sa caractéristique essentielle est la surrection de la plupart des reliefs des chaînes actuelles at l'existence de cisaillements importants. Les mouvements finimiocènes se sont poursuivis depuis mais le déroulement et la chronologie de ces mouvements sont encore mal connus.

Au Pliocène, la mer revient sur les marges et transgresse sur les continents actuels où elle forme des golfes et des bassins. Le caractère brutal de cette transgression, indiqué en particulier par le faciès profond des marnes bleues plaisanciennes qui recouvrent les évaporites, avait été souligné par Bourcart [4] et a été confirmé par les forages du Glomar Challenger (Cita, communication personnelle).

\section{La subsidence du bassin profond et le dépôt des évaporites}

Nous avons montré que le bassin algéro-provençal, au moins dans la partie située au Nord d'une ligne Sud Baléares-Sicile, s'était probablement ouvert à l'Oligocène moyen, postérieurement au paroxysme alpin, durant l'épisode de distension responable de la série de fossés découpant les blocs européen et sarde. Nous avons indiqué que la couche sédimentaire sur laquelle reposent les évaporites messiniennes n'est pas affectée tectoniquement. Il s'en suit que la configuration actuelle du bassin, y compris le sillon nord-africain, était acquise au plus tard à la fin du Miocène Inférieur et que la partie abyssale n'a depuis subi que des mouvements épéirogéniques de grande amplitude. Au total, plus de six kilomètres de sédiments s'y sont déposés depuis l'Oligocène moyen. Les marges continentales ont été le lieu de mouvements de découplage d'une amplitude d'au moins six kilomètres qui se sont nécessairement produits entre la partie abyssale et le continent adjacent. Elles ont donc été affectées par une tectonique verticale très importante durant tout le Cénozoïque Supérieur.

Ayant admis que la création du bassin s'était faite 


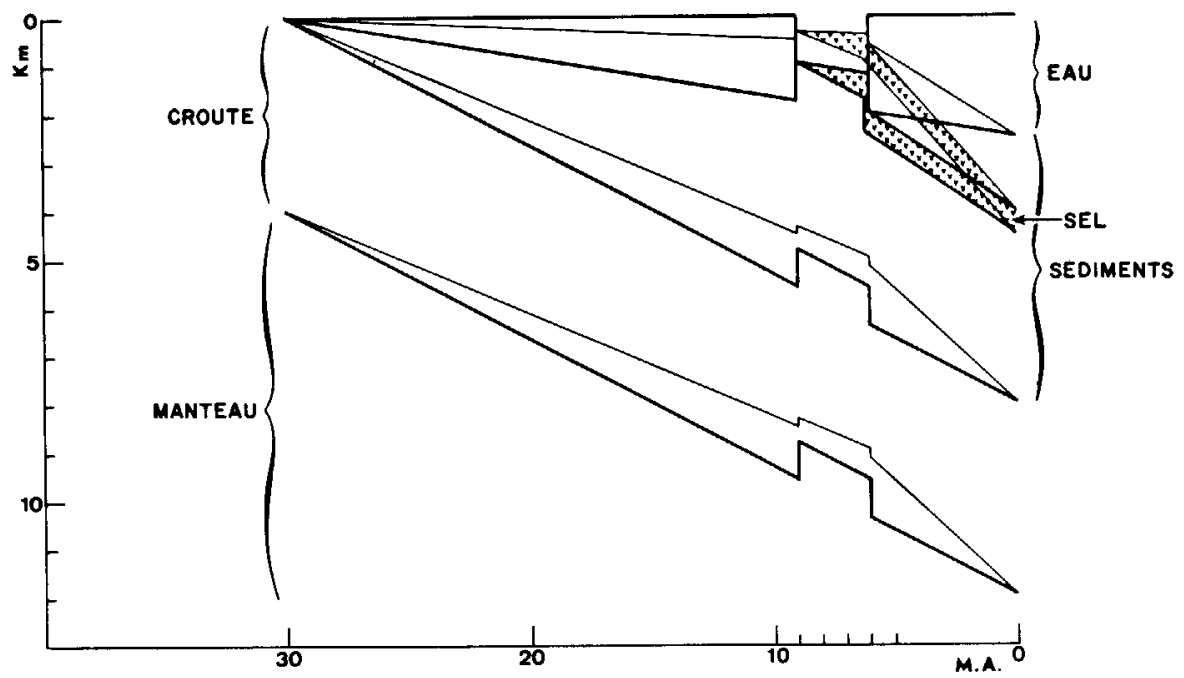

Fig. 3. Evolutions possibles de la subsidence du bassin de la Méditerranée occidentale depuis l'Oligocène moyen ( $30 \mathrm{~m} . \mathrm{a}$.). Deux cas possibles: en traits épais: subsidence constante; en traits fins: fort accroissement de la subsidence après le Messinien.

durant l'Oligocène moyen par un processus de dérive relative des blocs continentaux, il s'en suit que la subsidence du bassin a dû s'amorcer à cette époque. Le problème est de savoir quelle a été l'évolution de l'approfondissement du bassin en fonction de la combinaison des processus de subsidence et de sédimentation. En particulier, les évaporites messiniennes s'etant très probablement déposées sous une faible profondeur d'eau, il faut savoir si le niveau du bassin était comparable au niveau actuel ( $-2500 \mathrm{~m}$, hypothèse A, fig. 3 , traits épais, fig. 4A) ou s'il était à un niveau continental ( 0 à $-500 \mathrm{~m}$, hypothèse $\mathrm{B}$, fig. 3 , traits fins, fig. 4B). En effet, Ryan et Hsü (communication personelle) ont
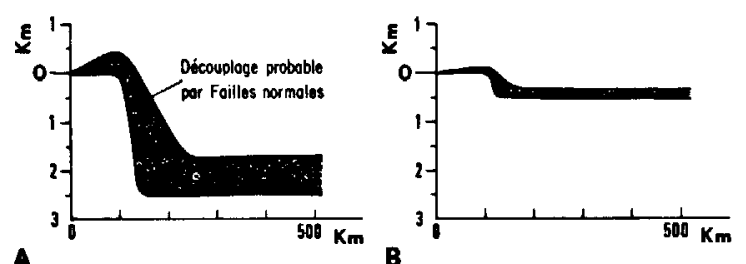

Fig. 4. Ajustement isostatique du bassin et des marges continentales après l'assèchement messinien: (1) Surface asséchée. (2) Surface ennoyée. A: protondeur d'eau $2,5 \mathrm{~km}$. On doit avoir un bombement du bord de la marge qui doit entraîner une érosion importante des couches pré-messiniennes. B: profondeur d'eau $0,5 \mathrm{~km}$. proposé un assèchement très important du bassin durant le Messinien pour expliquer le dépot des évaporites.

Cette hypothèse d'un abaissement très important du niveau de la mer avait originellement été proposée par Denizot [40] pour expliquer la présence d'évaporites messiniennes sous $1200 \mathrm{~m}$ de Pliocène marin dans le delta du Rhône.

La fig. 3 schématise l'évolution du phénomène de subsidence du bassin dans les deux hypothèses. On voit que des variations relatives assez faibles du taux de sédimentation par rapport au taux de subsidence peuvent entrainer des variations importantes de la profondeur du bassin. Nous admettons arbitrairement dans l'hypothèse $A$ une profondeur d'eau pré-Messinienne voisine de l'actuelle, c'est-à-dire $2500 \mathrm{~m}$, alors que cette profondeur d'eau était très faible à l'Oligocène. Admettre une profondeur de 1000 à $2000 \mathrm{~m}$ à l'Oligocène ne changerait pas le raisonnement que nous allons tenir.

Dans ce cas, la subsidence oligo-miocène est du même ordre que la subsidence post-messinienne. Le réajustement isostatique produit par l'assèchement du bassin entraine une remontée de la partie abyssale d'environ un tiers de sa profondeur $(800 \mathrm{~m})$. Le découplage le long des marges se traduit par un bombe- 
ment probable du bord du plateau durant le Messinien (fig. 4A). Le retour de l'eau se fait de manière brutale.

Il est remarquable que la couche évaporitique messinienne ne se trouve pas dans le bassin à moins de $2500 \mathrm{~m}$ de profondeur. On n'en voit pas de lambeaux plus haut sur les marges ou sur le plateau, comme dans le golfe du Lion par exemple. Lorsqu'elle est présente, en épaisseur importante, sur le continent, comme dans le bassin sicilien, il est évident qu'elle a depuis été surélevée. L'existence d'un faciès profond dans le Plaisancien, juste au-dessus des évaporites sans conglomérat ni lacune apparente, suggère que même dans les bassins maintenant continentaux les évaporites furent déposées dans un bassin profond de plus de $1000 \mathrm{~m}$. L'important épisode d'érosion qui affecte les marges au Messinien et qui est la cause du creusement des canyons s'explique bien, de même que la rapidité et la simultanéité de l'invasion marine. Par contre, l'existence d'une très vaste dépression asséchée de plus de $2000 \mathrm{~m}$ de profondeur durant le Messinien n'a pas d'analogue moderne.

Dans l'hypothèse $B$ (traits fins sur la fig. 3), la subsidence oligo-miocène est beaucoup plus faible que la subsidence post-messinienne. Il est probable que l'effondrement post-messinien du bassin se traduirait alors par une transgression progressive et pas entièrement synchronique sur le pourtour des marges. Dans ce cas, l'épisode évaporitique du Messinien a une signification tectonique importante, puisqu'il amène la subsidence brutale de l'ensemble de la Méditerranée occidentale de près de $2000 \mathrm{~m}$ à la fin du Messinien. Ceci serait en accord avec l'interprétation classique du début de la phase tectonique pontienne.

Par contre, dans l'hypothèse $A$, la signification tectonique de cet épisode est mineur puisqu'il entraîne uniquement des réajustements à un assèchement, puis à un nouveau remplissage du bassin. Pour notre part, nous penchons pour l'hypothèse $A$, dont le mécanisme peut assez facilement s'expliquer. En effet, la fermeture tectonique des détroits entraîne un déséquilibre entre le bilan d'évaporation et d'apport d'eau. A l'heure actuelle, une fermeture du déroit de Gibraltar (qui ne fait que $420 \mathrm{~m}$ de profondeur) provoquerait un assèchement de la Méditerranée en quelques milliers d'années *.

Remarquons que, si cette hypothèse est exacte, il faut prendre grand soin de distinguer entre les mouve- ments verticaux dus aux réajustements isostatiques et les mouvements orogéniques possibles. En particulier n'est-il pas possible qu'une partie des phénomènes de distension du Miocène supérieur attribués jusqu'ici à une phase orogénique, soient en fait le résultat de réajustements isostatiques autour de la Méditerranée à la suite de cet épisode d'assèchement ? Ceci pourrait réconcilier les données géologiques avec les données océaniques qui indiquent une phase de compression pure dans cette région depuis au moins 9 M.a. [21]

Enfin, dans cette hypothèse, on doit avoir à la même époque un assèchement de la Méditerranée orientale, aussi bien que de la Mer Rouge, dans la mesure où cette dernière ne communiqua avec l'Océan Indien qu'au Pliocène. Or on sait que des dépôts évaporitiques Messiniens existent en Méditerranée orientale, aussi bien sur les bordures que dans le domaine profond. De même, en Mer Rouge, les dépôts évaporitiques débutant au Miocène inférieur se sont poursuivis jusqu'au Messinien compris.

\section{Remerciements}

Messieurs Durand-Delga, Mattauer et Thaler ont lu et critiqué une première version du manuscrit et leurs remarques nous ont été très utiles.

\section{Références}

[1] L. Montadert, S. Sancho, J. P. Debyser et E. Winnock, De l'âge tertiaire de la série salifère responsable des structures diapiriques en Méditerranée occidentale, $\mathbf{C}$. R. Acad. Sc., Paris, 271 (1970) 812.

[2] J. M. Auzende, J. Bonnin, J. L. Olivet, G. Pautot et A. Mauffret, Upper Miocene salt layer in the western Mediterranean basin, Nature 230 (1971) 82.

[3] K. J. Hsu, Conférence de presse, Paris, octobre 1970.

[4] J. Bourcart, La Méditerranée et la révolution du Pliocène, dans: Livre à la mémoire du Professeur Fallot, Mem. h. Ser. Soc. Géol. France, 1 (1962) 103.

[5] L. Glangeaud, Les méthodes de la géodynamique et leur application aux structures de la Méditerranée occidentale, Rev. Géog. Phys. Géol. Dynam. X, 2 (1968) 83.

- Le volume d'eau mediterranéen est de $4,238,000 \mathrm{~km}^{3}$ et le bilan d'entrée sur sortie à Gibraltar est de $1500 \mathrm{~km}^{3} / \mathrm{an}$ [39]. Il faudrait donc théoriquement un peu plus de 2600 ans. 
[6] J. Aubouin et G. Mennessier, Essai sur la structure de la Provence, dans: Livre à la mémoire du Professeur Fallot, Mem. h. Ser. Soc. Géol. France, 1 (1962) 45

[7] D. A. Fahlquist et J. B. Hersey, Seismic refraction measurements in the western Mediterranean sea, Bull. Inst. Océanog. Monaco, 67 (1969) $52 \mathrm{pp}$.

[8] M. J. Berry et L. Knopoff, Structure of the upper mantle under the western Mediterranean basin, $\mathbf{J}$. Geophys. Res. 14 (1967) 3613.

[9] E. Le Borgne, J. Le Mouel et X. Le Pichon, Aeromagnetic survey of south western Europe and plate tectonics (en préparation).

[10] L. Glangeaud, Paléogéographie dynamique de la Méditerranée et de ses bordures. Le róle des phases pontoplio-quatemaire, dans: Colloque du C.N.R.S. Villefranche-sur-Mer (1961) 125

[11] W. B. F. Ryan. The floor of the Mediterranean Sea, Ph. D. Thesis, Columbia University (1969).

[12] J. Bourcart, Morphologie du Précontinent des Pyrénées à la Sardaigne, dans: Coll. Int. C.N.R.S. Paris (1959).

[13] G. Dubourdieu, Le déplacement de l'Europe occidentale, C. R. Acad. Sci. Paris 254 (1962) 510.

[14] D. J. Stanley et E. Mutti. Sedimentological evidence for an emerged land mass in the Ligurian Sea during the Palaeogene, Nature 218 (1968) 32 .

[15] A. J. Pannekoek. Uplift and subsidence in and around the Western Mediterranean since the Oligocene: a review, Verhandelingen Kon. Ned. Geol. Mijnbouw. gen. 26 (1969) 53.

[16] J. Flandrin, Contribution à l'étude stratigraphique du Nummulitique algérien, Bull. Serv. Cart Géol. Algérie, lére S., no 8 (1948).

[17] P. H. Kuenen, L'age d'un bassin méditerranéen, dans: Coll. Int. C.N.R.S., Paris (1959).

[18] W. S. Carey, The Orocline concept in geotectonics, Proc. Roy. Soc. Tasman, 89 (1958) 177

[19] X. Le Pichon, J. Bonnin, J. Francheteau et J. C. Sibuet, Une hypothèse d'évolution tectonique du golfe de Gascogne, dans: Coll. sur le golfe de Gascogne. C.N.E.X.O.. I.F.P., Rueil-Malmaison (1970) à paraître.

[20] J. Cogne, B. Geze, J. Goguel, J. Grolier, J. Letourneur, J. Pellet, J. Rothe et C. Sitter, Les rifts et les failles de décrochement en France, Rev. Geogr. Phys. Géol. Dynam. (2) VIII (1969) 123.

[21] W. C. Pitman III, M. Talwani and J. R. Heirtzler, Age of the North Atlantic Ocean from magnetic anomalies, Earth Planet. Sci. Letters 11 (1971) 195.

[22] C. Cornet, La Provence de YOligocène à nos jours, Rev. Géogr. Phys. Géol. Dynam. (2) XI (1969) 101.

[23] R. Delcey, J. C. Limasset et P. Routhier, Les bassins sédimentaires du Nord de la Corse: essai de synthèse stratigraphique et aperçu tectonique, Bull. Soc. Géol. France (7) VI (1964) 324.

[24] G. Pautot, J. M. Auzende et X. Le Pichon, Continuous deep salt layer along north Atlantic margins related to early phase of rifting, Nature 227 (1970) 351.

[25] B. C. Heezen, J. Univ. Missouri at Rolla 1 (1968) 5.

[26] R. Anglada, Sur la position du datum à globigerinoides (Foraminiferida), la zone Nu (Blow, 1967) et la limite oligo-miocène $t n$ Méditerranée, C. R. Acad. Sci. Paris 1971 (1967) 272.

[27] A. Caire, Morphotectonique de l'Autochtone présaharien et de l'allochtone tellien. Rev. Géogr. Phys. Géol. Dynam. (3) VII (1965) 267

[28] M. Durand-Delga et M. Mattauer, Sur l'origine ultrarifaine de certaines nappes du $R$ if septentrional. C. R. Somm. Soc. Geol. France (1960) 22.

[29] M. Kicken, Les traits essentiels de la géologie algérienne, dans: Livre à la mémoire du Professeur Fallot, Mem. h. Ser. Soc. Géol. France 1 (1962) 545.

[30] J. M. Auzende et G. Pautot, La marge continentale algérienne et le phénomène de subsidence: exemple du golfe de Bougie, C. R. Acad. Sci. Paris 271 (1970) 1945

[31] M. Durand-Delga. Structure and Geology of the North East Atlas Mountains. Guide book to the geology and history of Tunisia, Petrol. Expl. Soc. Lybia, 9th Ann. Meeting Field Conf., Tripoli, ed. Levis Martini (1967).

[32] F. Wezel, Numidian Flysch: an Oligocene-early Miocene continental rise deposit off the African platform, Nature 228 (1970) 275.

[33] A. Perrodon, Etudes géologiques des bassins néogènes sublittoraux de l'Algérie occidentale, Publ. Serv. Carte Géol. Algérie 12 (1957).

[34] L. Ogniben, Nota illustrativa dello schema geologico della Sicila nord-orientale, Riv. Min. Siciliana 11 64-65 (1960) 183.

[35] L. Glangeaud, B. Schlich, G. Pautot, G. Bellaiche, P. Patriat et M. Ronfard, Morphologie, tectonophysique et évolution géodynamique de la bordure sous-marine des Maures et de l'Estérel. Relations avec les régions voisines, Bull. Soc. Géol. France 7 VI (1965) 998.

[36] B. Geze, Caractères structuraux de l'Arc de Nice (AlpesMaritimes), dans: Livre à la mémoire du Professeur Fallot, Mem. h. Ser. Soc. Géol. France, II (1960) 289.

[37] L. Glangeaud, Les nouvelles théories sur la formation du Jura, Bull. Soc. Hist. Nat. Doubs 52 (1948) 5.

[38] J. Debelmas et M. Lemoine, The western Alps. Palaeogeography and structure, Earth Sci. Rev. 6 (1970) 221.

[39] H. Lacombe et P. Tchernia, Quelques considérations sur le régime du détroit de Gibraltar d'après les mesures effectuées en septembre 1960, Ext. Rap. Proc. Verb. des réunions de la CIESMM. XVII (3) (1963).

[40] G. Denizot, Les anciens rivages de la Méditerranée française. Bull. Inst. Ocean. Monaco 992 (1951) 56 pp. 